\title{
Spectrométrie parTF dans les VUV. Perspectives offertes par des interféromètres sans lames séparatrices
}

F. Polack et D. Joyeux*

Lure, Bâtiment 209D, Centre Universitaire, 91406 Orsay cedex, France

* Institut d'Optique, Bâtiment 503, Centre Universitaire, BP. 147, 91403 Orsay cedex, France

\begin{abstract}
Résumé : La spectrométrie par transformation de Fourier peut être étendue au domaine des VUV et même des XUV par l'emploi d'interféromètres à division de front d'onde qui n'utilisent pas de lame séparatrice. Les sources synchrotrons de troisième génération permettent d'obtenir la cohérence spatiale nécessaire à leur bon fonctionnement. Un projet d'interféromètre par TF VUV est décrit et ses performances potentielles sont examinées.
\end{abstract}

\section{INTRODUCTION}

Dans le visible et le proche UV les spectromètres par TF (transformation de Fourier), permettent d'atteindre des résolutions très élevées, jusqu'à $10^{6}$. Ils sont basés sur des interféromètres, dits à division d'amplitude, qui exigent l'emploi d'une lame séparatrice. La réalisation de cette séparatrice pose problème dans le domaine des VUV et plus encore des XUV par manque de matériaux transparents pour les supports comme pour les couches réfléchissantes[1,2]. Force est donc d'employer des instruments à réseaux dont la résolution peut atteindre $10^{5}$ dans l'UV proche, mais décroît avec l'énergie de photon et ne dépasse pas quelques $10^{4}$ vers $60 \mathrm{eV}$.

Cette résolution est insuffisante pour certaines applications : dans les VUV, la mesure des sections efficaces absolues de raies d'absorption a d'importantes implications en physique moléculaire et en astrophysique mais demande des résolutions supérieures à $10^{5}$; dans les XUV une résolution de $10^{5}$ au moins est nécessaire pour valider les modèles de double ionisation de l'hélium et des ions héliumoïdes. Ces problèmes justifient le développement de spectromètres par TF pour les courtes longueurs d'onde[2].

\section{UN INTERFEROMETRE POUR LA SPECTROSCOPIE PAR TF XUV}

Pour la spectrométrie par TF il convient de réaliser un interféromètre à différence de marche variable $x$. L'échantillon à mesurer est placé dans le trajet commun des faisceaux. On enregistre l'état d'interférence en fonction de $x$, c'est l'interférogramme. A partir de cet interférogramme, la transmittance spectrale de l'échantillon peut être calculée par transformée de Fourier inverse. Pour obtenir une résolution spectrale $R$, il est nécessaire d'enregistrer l'interférogramme jusqu'à une différence de marche $x=R \lambda$. Le théorème d'échantillonnage exige en principe l'acquisition de $R$ échantillons, mais l'utitisation d'un pré-monochromateur de bande passante 1/100 par exemple, autorise à réduire le nombre d'échantillons par ce même facteur. 
Dans les VUV et jusque dans les $\mathrm{X}$ mous on peut utiliser des interféromètres à division de front d'onde qui, tel l'interféromètre à miroirs de Fresnel, n'emploient pas de séparatrice[3]. Leur principe consiste à réfléchir différemment et à faire interférer deux portions de la surface d'onde émise par la source, il exige donc une certaine cohérence spatiale de celle-ci. Pour l'obtenir il suffit de sélectionner une étendue géométrique étroite de l'ordre de $\lambda^{2}$. Des sources de rayonnement synchrotron comme Super-ACO à LURE, permettent d'obtenir un flux suffisant dans cette étendue, et les sources synchrotrons les plus récentes, dites de troisième génération, sont quasi cohérentes à ces longueurs d'onde.

La figure 1 illustre une façon de réaliser un interféromètre à différence marche variable en n'employant que des miroirs plans (le plan est la surface qui peut être polie avec la meilleure précision). La figure 2 schématise la façon envisagée de contrôler la translation et le parallélisme des miroirs.

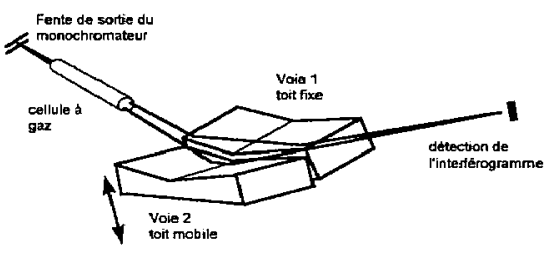

Figure1 : Interféromètre à réflecteurs en toit

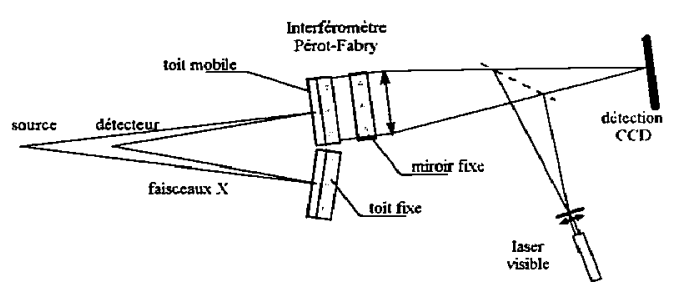

Figure 2 : Principe du contrôle de l'interféromètre

\section{PARAMETRES ET PERFORMANCES}

On utilise des réflecteurs en toit pour leur capacité à conserver la position du faisceau émergent au cours de la translation. L'angle d'incidence et l'angle du toit dépendent de l'énergie de photon. Pour illustrer le concept, voici les paramètres permettant de travailler autour de $20 \mathrm{eV}$. L'angle d'incidence sera $45^{\circ}$, soit un angle de toit de $90^{\circ}$, et le déplacement du toit pour $R=510^{5}$ sera de $15 \mathrm{~mm}$. Les défauts de fabrication et d'alignement des miroirs devront être inférieurs à $\lambda / 400$ (visible) et la position des échantillons précise à $\lambda / 250$. Le flux estimé sur un onduleur à Super-ACO est de $10^{10}$ photons $\mathrm{s}^{-1}$ dans $\Delta \lambda / \lambda=10^{-2}$, ce qui doit permettre d'acquérir un spectre de $10^{3}$ points en 45 minutes.

\section{CONCLUSION}

La construction d'un interféromètre pour la spectrométrie TF est un objectif réaliste à Super-ACO pour les VUV. Sur une machine comme le futur anneau SOLEIL le domaine d'énergie d'un tel spectromètre pourrait être étendu jusqu'au X mous.

\section{Références}

[1] Yoshino K., Smith P.L., Parkinson W.H., Thorne A.P. et Ito K., Rev. Sci. Instrum. 66 (1995) 2122-2124

[2] Howells M.R., Franck K., Hussain Z., Moler E.J., Reich T., Möller D. et Shirley D.A., Nucl. Instrum. Methods A 347 (1994) 182-191

[3] Polack F., Joyeux D., Svatoš J. et Phalippou D., Rev. Sci. Instrum. 66 (1995) 2180 2183 